\title{
High-velocity interstellar clouds towards the M 15 globular cluster
}

\section{Low resolution optical data}

\author{
N. Lehner ${ }^{1}$, W.R.J. Rolleston ${ }^{1}$, R.S.I. Ryans ${ }^{1}$, F.P. Keenan ${ }^{1}$, B. Bates ${ }^{1}$, D.L. Pollacco ${ }^{2}$, and K.R. Sembach ${ }^{3}$ \\ 1 Department of Pure and Applied Physics, The Queen's University of Belfast, Belfast, BT7 1NN, Northern Ireland \\ 2 Isaac Newton Group, Apartado de Correos 368, Santa Cruz de La Palma, Tenerife 38780, Canary Islands, Spain \\ 3 The John Hopkins University, Department of Physics and Astronomy, Bloomberg Center, 3400 N. Charles Street, Baltimore \\ MD 21218, U.S.A.
}

Received July 10; accepted August 13, 1998

\begin{abstract}
We present low resolution interstellar spectra of the CaII K and NaI D lines towards 12 stars in the M 15 globular cluster. These sightlines are used to study the small scale structure of the interstellar medium, over scales of a few arcseconds to a few arcminutes. A highvelocity cloud is detected in the $\mathrm{Ca}$ II and $\mathrm{Na}$ I lines at a LSR velocity of approximately $\sim 70 \mathrm{~km} \mathrm{~s}^{-1}$. Tentative velocity and equivalent width variations are observed on scales of a few arcseconds; however, higher resolution data are necessary to quantify the magnitude of these variations. We discuss these observations and their importance for future studies of this high velocity gas at high spectral resolution.
\end{abstract}

Key words: ISM: individual objects: high-velocity clouds - ISM: general - ISM: clouds - ISM: structure - globular cluster: individual: M 15 - stars: early-type

\section{Introduction}

This article is a continuation of studies using radio $\mathrm{HI}$, optical and ultraviolet observations (Little et al. 1994; Sembach 1995; Ryans et al. 1996; Kennedy et al. 1998) of a high-velocity cloud (HVC) observed towards both the M 15 globular cluster (Galactic co-ordinates $l, b \sim 65.0^{\circ}$, $-27.3^{\circ}, d \sim 10.4 \mathrm{kpc}$; Durrel \& Harris 1993), and the nearby $\left(2.5^{\circ}\right.$ away) blue halo star HD 203664 ( $d \sim 3.2 \mathrm{kpc}$; Little et al. 1994).

Little et al. (1994) detected the HVC at a Local Standard of Rest (LSR) velocity $v_{\mathrm{LSR}} \simeq 80 \mathrm{~km} \mathrm{~s}^{-1}$, using both $\mathrm{HI}$ and optical absorption line observations. Further high-resolution optical spectroscopy of this sightline revealed the presence of multiple cloud components at $v_{\mathrm{LSR}} \simeq 70,75,80 \mathrm{~km} \mathrm{~s}^{-1}$ (Ryans et al. 1996). The HVC

Send offprint requests to: N. Lehner; N.Lehner@qub.ac.uk emission is weak (brightness temperature $T_{\mathrm{b}} \sim 0.07 \mathrm{~K}$ ), which makes detailed comparisons between the $\mathrm{HI}$ emission and optical absorption line structure of the HVC difficult. However, much stronger HVC emission at similar velocities $\left(T_{\mathrm{b}} \sim 1.3 \mathrm{~K}\right)$ is present across the $\mathrm{M} 15$ globular cluster, and initial inspection of $\mathrm{NaI}$ absorption profiles towards stars in this direction show that the HVC gas lies in front of the cluster (Kennedy et al. 1998). Therefore, cluster members of M 15 provide an unprecedented opportunity to probe the properties within the HVC over small spatial scales.

Hot OB-type stars provide the best background objects to study interstellar features, since they possess clean spectra with few stellar lines, and display well defined continua. In this paper we report a survey to identify hot stars in M 15 that show strong HVC absorption in the Na I $\mathrm{D}_{1} \&$ $\mathrm{D}_{2}$ and CaII $\mathrm{K}$ features. These stars are therefore suitable for further high resolution studies of this cloud.

\section{Observations and data reduction}

\subsection{The sample}

The programme stars were chosen from the $U B V$ photometry of Buonanno et al. (1983) and Battistini et al. (1985), using the following selection criteria:

- Stars should be B-type, to provide suitable continua and relatively clean spectra with few stellar lines. Hence, assuming a reddening, $E(B-V) \sim 0.1$ (Buonanno et al. 1983) towards M 15, we have selected all objects with $B-V \lesssim 0.1$.

- Stars should be sufficiently bright to allow high resolution, high signal-to-noise spectra to be obtained using $4 \mathrm{~m}$ class telescopes. We have imposed a faint magnitude limit $V=17$. This criterion excludes the fainter sdO/early sdB stars; hence, our targets mainly comprise blue horizontal branch (BHB) and PostAsymptotic Giant Branch (PAGB) objects. 
Table 1. Sample of stars in the nearby field of M 15 used to search for HVC absorption

\begin{tabular}{lcrcc}
\hline Star & $\begin{array}{c}V \\
(\mathrm{mag})\end{array}$ & $\begin{array}{c}B-V \\
(\mathrm{mag})\end{array}$ & $\begin{array}{c}l \\
\left({ }^{\circ}\right)\end{array}$ & $\begin{array}{c}b \\
\left({ }^{\circ}\right)\end{array}$ \\
\hline & & & & \\
$\mathrm{K}-027$ & 16.6 & -0.03 & 65.083 & -27.294 \\
$\mathrm{~K}-048$ & 17.0 & -0.01 & 65.050 & -27.272 \\
$\mathrm{~K}-064$ & 15.9 & 0.08 & 65.053 & -27.283 \\
$\mathrm{~K}-080$ & 16.0 & 0.09 & 65.080 & -27.319 \\
$\mathrm{~K}-084$ & 16.5 & 0.00 & 65.052 & -27.289 \\
$\mathrm{~K}-127^{a}$ & 16.0 & 0.13 & 65.073 & -27.320 \\
$\mathrm{~K}-143$ & 16.4 & 0.03 & 65.049 & -27.297 \\
$\mathrm{~K}-154$ & 16.8 & -0.08 & 65.057 & -27.307 \\
$\mathrm{~K}-239$ & 14.3 & 0.09 & 65.055 & -27.330 \\
$\mathrm{~K}-258$ & 16.5 & 0.06 & 65.056 & -27.336 \\
$\mathrm{~K}-267$ & 16.7 & 0.03 & 65.051 & -27.334 \\
$\mathrm{~N}-1^{b}$ & - & - & 65.044 & -27.290 \\
\hline$a:$ Not previously a target.
\end{tabular}

$a$ : Not previously a target.

$b$ : Previously unidentified star.

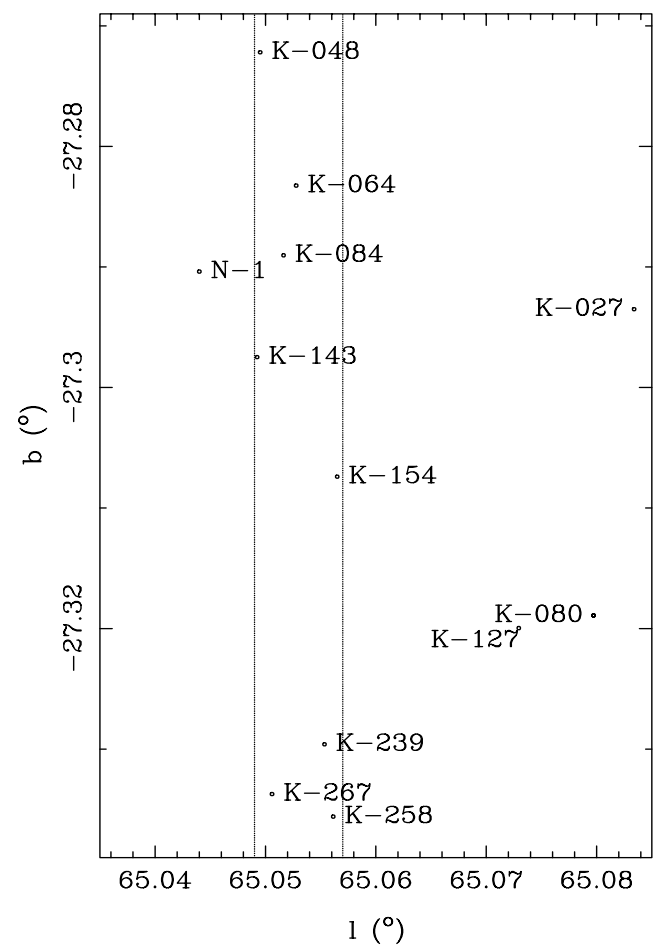

Fig. 1. Programme stars observed in the globular cluster M 15. Stars within the region enclosed by the dotted lines have their equivalent widths and radial velocities plotted in Fig. 4

- Stars should also be members of the M 15 globular cluster. Foreground stars were eliminated by identifying objects with stellar radial velocities that were not consistent with the nominal cluster velocity (Dull et al. 1997).

- Finally, priority was given to stars with separations of less than $1^{\prime}$ to allow studies on small spatial scales.

Table 1 lists the positions and magnitudes of stars that were found to meet these criteria, and their positions are plotted in Fig. 1. The star identifications in Table 1 correspond to those in Table II of Buonanno et al. (1983) and Battistini et al. (1985). Observations of K-127 were serendipitously obtained with K-080, and N-1 with K-143 and $\mathrm{K}-154$.

\subsection{ISIS observations of cluster stars}

The observations were carried out using the ISIS spectrograph on the $4.2 \mathrm{~m}$ William Herschel Telescope at La Palma Observatory on 16/17 August 1997. The blue and red arms were operated with the $\mathrm{H} 2400 \mathrm{~B}$ lines/mm grating (Loral $2048 \times 2048 \mathrm{CCD}$ ) and R1200R lines/mm grating (Tek $1024 \times 1024$ CCD), respectively. The spectra were centered on $3960 \AA$ for the Ca II K and H lines (blue arm) and $5900 \AA$ for the Na I D lines (red arm). This instrumental configuration resulted in a FWHM velocity resolution of $\sim 42 \mathrm{~km} \mathrm{~s}^{-1}$ for the Na I data and $\sim 30 \mathrm{~km} \mathrm{~s}^{-1}$ for the $\mathrm{Ca}$ II data, determined from $\mathrm{CuAr}$ arc lines used for wavelength calibration. The slit width was 1 ". 03 , with a scale of 0 ". 21 per pixel for the blue arm and $0^{\prime \prime} 33$ per pixel for the red arm. Multiple exposures were made for each star, interspersed with $\mathrm{CuAr}$ arc line calibration exposures. Bias and flat field exposures were made at the start and the end of each night.

\subsection{Data reduction}

The raw CCD images were reduced using standard techniques within the NOAO IRAF package (Morris \& Privett 1995). The spectra were wavelength calibrated, and crosscorrelation techniques were employed for multiple spectra of the same object to ensure that there was no shift between successive exposures. Images were then co-added, using weights determined by their continuum signal-tonoise levels. Typically, co-added spectra have a signal-tonoise ratio $(\mathrm{S} / \mathrm{N})$ of about 50 for the red data, and in the range $7-20$ for the blue data. The spectra were then shifted to the Dynamical LSR (Mihalas \& Binney 1981), using corrections obtained from the STARLINK RV (Wallace \& Clayton 1996) package.

Although the CaII $\mathrm{K}$ and $\mathrm{H}$ lines are free of telluric absorption features, the Na I D lines show evidence of contamination. However, a comparison of a synthetic telluric spectrum with the observational data demonstrated that only the continuum level was affected, and that the telluric lines would not affect the measurement of HVC radial velocities. Therefore we did not remove these telluric lines from the data.

\section{Analysis and results}

\subsection{Line profile fitting}

Interstellar Ca II $\mathrm{K}$ and $\mathrm{Na}$ I $\mathrm{D}_{1} \& \mathrm{D}_{2}$ features were modelled using Gaussian fitting routines in the STARLINK package DIPSO (Howarth et al. 1996) to obtain radial velocity 
Table 2. Equivalent width and radial velocity data for both the stellar and interstellar Ca II K lines

\begin{tabular}{lccrcrcrc}
\hline \multicolumn{2}{c}{ Sightline } & \multicolumn{7}{c}{ Interstellar (IS) } \\
\hline Star & $\mathrm{S} / \mathrm{N}$ & $\begin{array}{c}W_{\star} \\
(\AA)\end{array}$ & $\begin{array}{c}v_{\star} \\
\left(\mathrm{km} \mathrm{s}^{-1}\right)\end{array}$ & $\begin{array}{c}W_{\text {LIS }} \\
(\AA)\end{array}$ & $\begin{array}{c}v_{\text {LIS }} \\
\left(\mathrm{km} \mathrm{s}^{-1}\right)\end{array}$ & $\begin{array}{c}W_{\mathrm{HVC}} \\
(\AA)\end{array}$ & $\begin{array}{c}v_{\mathrm{HVC}} \\
\left(\mathrm{km} \mathrm{s}^{-1}\right)\end{array}$ & $\begin{array}{c}v_{\text {rel }} \\
\left(\mathrm{km} \mathrm{s}^{-1}\right)\end{array}$ \\
\hline $\mathrm{K}-027$ & 7 & $0.17 \pm 0.05$ & $-89 \pm 2$ & $0.27 \pm 0.09$ & $-3 \pm 4$ & $0.10 \pm 0.05$ & $59 \pm 3$ & 62 \\
$\mathrm{~K}-048$ & 7 & $0.23 \pm 0.09$ & $-90 \pm 2$ & $0.24 \pm 0.07$ & $-6 \pm 5$ & $0.15 \pm 0.06$ & $69 \pm 3$ & 75 \\
$\mathrm{~K}-064$ & 10 & $0.25 \pm 0.07$ & $-96 \pm 7$ & $0.25 \pm 0.12$ & $-6 \pm 2$ & $0.16 \pm 0.06$ & $64 \pm 3$ & 70 \\
$\mathrm{~K}-080$ & 12 & $0.26 \pm 0.08$ & $-94 \pm 3$ & $0.21 \pm 0.07$ & $-7 \pm 3$ & $0.07 \pm 0.06$ & $57 \pm 4$ & 64 \\
$\mathrm{~K}-084$ & 11 & $0.43 \pm 0.08$ & $-97 \pm 2$ & $0.38 \pm 0.08$ & $-8 \pm 2$ & $0.05 \pm 0.05$ & $60 \pm 3$ & 68 \\
$\mathrm{~K}-127$ & 11 & $0.43 \pm 0.08$ & $-91 \pm 2$ & $0.32 \pm 0.08$ & $-9 \pm 2$ & $0.08 \pm 0.05$ & $65 \pm 2$ & 74 \\
$\mathrm{~K}-143$ & 12 & $0.10 \pm 0.06$ & $-102 \pm 4$ & $0.32 \pm 0.07$ & $-9 \pm 2$ & $0.09 \pm 0.06$ & $61 \pm 3$ & 70 \\
$\mathrm{~K}-154$ & 9 & $0.16 \pm 0.06$ & $-92 \pm 4$ & $0.28 \pm 0.08$ & $-4 \pm 3$ & $0.20 \pm 0.08$ & $66 \pm 5$ & 70 \\
$\mathrm{~K}-239$ & 9 & $0.12 \pm 0.05$ & $-93 \pm 1$ & $0.25 \pm 0.07$ & $-2 \pm 2$ & $0.13 \pm 0.05$ & $63 \pm 2$ & 65 \\
$\mathrm{~K}-258$ & 11 & $0.13 \pm 0.05$ & $-92 \pm 2$ & $0.27 \pm 0.07$ & $+10 \pm 2$ & $0.10 \pm 0.05$ & $80 \pm 3$ & 70 \\
$\mathrm{~K}-267$ & 8 & $0.11 \pm 0.08$ & $-88 \pm 7$ & $0.31 \pm 0.08$ & $-1 \pm 4$ & $0.07 \pm 0.05$ & $80 \pm 4$ & 79 \\
$\mathrm{~N}-1$ & 22 & $1.10 \pm 0.10$ & $-120 \pm 2$ & $0.27 \pm 0.07$ & $-3 \pm 2$ & $0.10 \pm 0.05$ & $63 \pm 3$ & 66 \\
\hline
\end{tabular}

Note: LIS refers to the local interstellar gas. Uncertainties are fitting errors at $1 \sigma$.

Table 3. Radial velocity data for the interstellar Na I D lines

\begin{tabular}{|c|c|c|c|}
\hline Star & $\begin{array}{c}v_{\text {LIS }} \\
\left(\mathrm{km} \mathrm{s}^{-1}\right)\end{array}$ & $\begin{array}{c}v_{\mathrm{HVC}} \\
\left(\mathrm{km} \mathrm{s}^{-1}\right)\end{array}$ & $\begin{array}{c}v_{\text {rel }} \\
\left(\mathrm{km} \mathrm{s}^{-1}\right)\end{array}$ \\
\hline \multicolumn{4}{|c|}{$\mathrm{NaI} \mathrm{D}_{1}$} \\
\hline $\mathrm{K}-080$ & $-11 \pm 4$ & $54 \pm 8$ & 65 \\
\hline K-084 & $-1 \pm 5$ & $66 \pm 2$ & 65 \\
\hline $\mathrm{K}-127$ & $-15 \pm 4$ & $47 \pm 15$ & 62 \\
\hline $\mathrm{K}-258$ & $+11 \pm 3$ & $80 \pm 6$ & 69 \\
\hline \multicolumn{4}{|c|}{$\mathrm{NaI} \mathrm{D}_{2}$} \\
\hline K-080 & $-16 \pm 3$ & $54 \pm 2$ & 70 \\
\hline K-084 & $+1 \pm 4$ & $65 \pm 4$ & 64 \\
\hline $\mathrm{K}-127$ & $-13 \pm 5$ & $45 \pm 14$ & 58 \\
\hline $\mathrm{K}-258$ & $+16 \pm 5$ & $78 \pm 5$ & 62 \\
\hline
\end{tabular}

Note: LIS refers to the local interstellar gas.

Uncertainties are fitting errors at $1 \sigma$.

and equivalent width measurements. The results are summarised in Tables 2 and 3, and the observed spectra and corresponding fits are displayed in Figs. 2 and 3. The lower resolution of the red spectra precluded the measurements of reliable equivalent widths for the $\mathrm{NaI} \mathrm{D}_{1} \& \mathrm{D}_{2}$ features, whilst CaII $\mathrm{H}$ is severely blended with stellar $\mathrm{HI}$ and He I lines; hence, these data are not discussed further. Additionally, due to the resolution of the ISIS spectra, we did not attempt to derive column densities and $b$-values for the HVC components.

\subsection{Cluster membership}

The stellar Ca II K line was used to determine the radial velocities of the target stars. Table 2 shows that these stars have blue-shifted velocities in the range $v_{\mathrm{LSR}}=-90$ to $-100 \mathrm{~km} \mathrm{~s}^{-1}$ which confirm their cluster membership (see, for example, Dull et al. 1997). We note that K-239, which is much brighter than our other targets, is most probably a PAGB star (see Durrel \& Harris 1993), while K-084 exhibits strong stellar Na I D absorption (see Fig. 3) and possesses a spectrum compatible with an early A-type star.

\subsection{Identification of $H V C$ absorption}

The sightlines show the HVC absorption in CaII K at velocities $v_{\mathrm{HVC}} \sim 60-80 \mathrm{~km} \mathrm{~s}^{-1}$ with equivalent widths that vary from 0.05 to $0.20 \AA$. However LSR velocities as high as $\pm 10 \mathrm{~km} \mathrm{~s}^{-1}$ for the local gas may be evidence for an incorrect assignment of the wavelength scale. Hence, in Tables 2 and 3 we also quote the difference, $v_{\text {rel }}$, between the measured velocity of the HVC and the low velocity interstellar gas. The adoption of either $v_{\mathrm{HVC}}$ or $v_{\text {rel }}$ in the subsequent analysis does not affect our principal conclusions (see Sect. 4).

The HVC was only detected in Na I D lines for four objects, and (as explained previously) only cloud velocities were reliably measured due to the low resolution of the red arm data. Within the limits of the uncertainties, there is a good agreement between the radial velocities measured for the HVC from Ca II and Na I lines (see Tables 2 and 3). Additionally, these radial velocities are in good agreement with values obtained from optical spectra and radio $\mathrm{H}$ I data by Langer et al. (1990) and Kennedy et al. (1998) towards the globular cluster M 15. Their observations were on a larger spatial scale than the present one, but show the same range in velocities.

\section{Discussion}

Our primary motivation in obtaining these spectra was to study the HVC towards M 15 on a small angular scale, and identify suitable stars for further studies at high spectral resolution. In Fig. 4 we have plotted the HVC equivalent widths and velocities versus the galactic latitude $b$ 

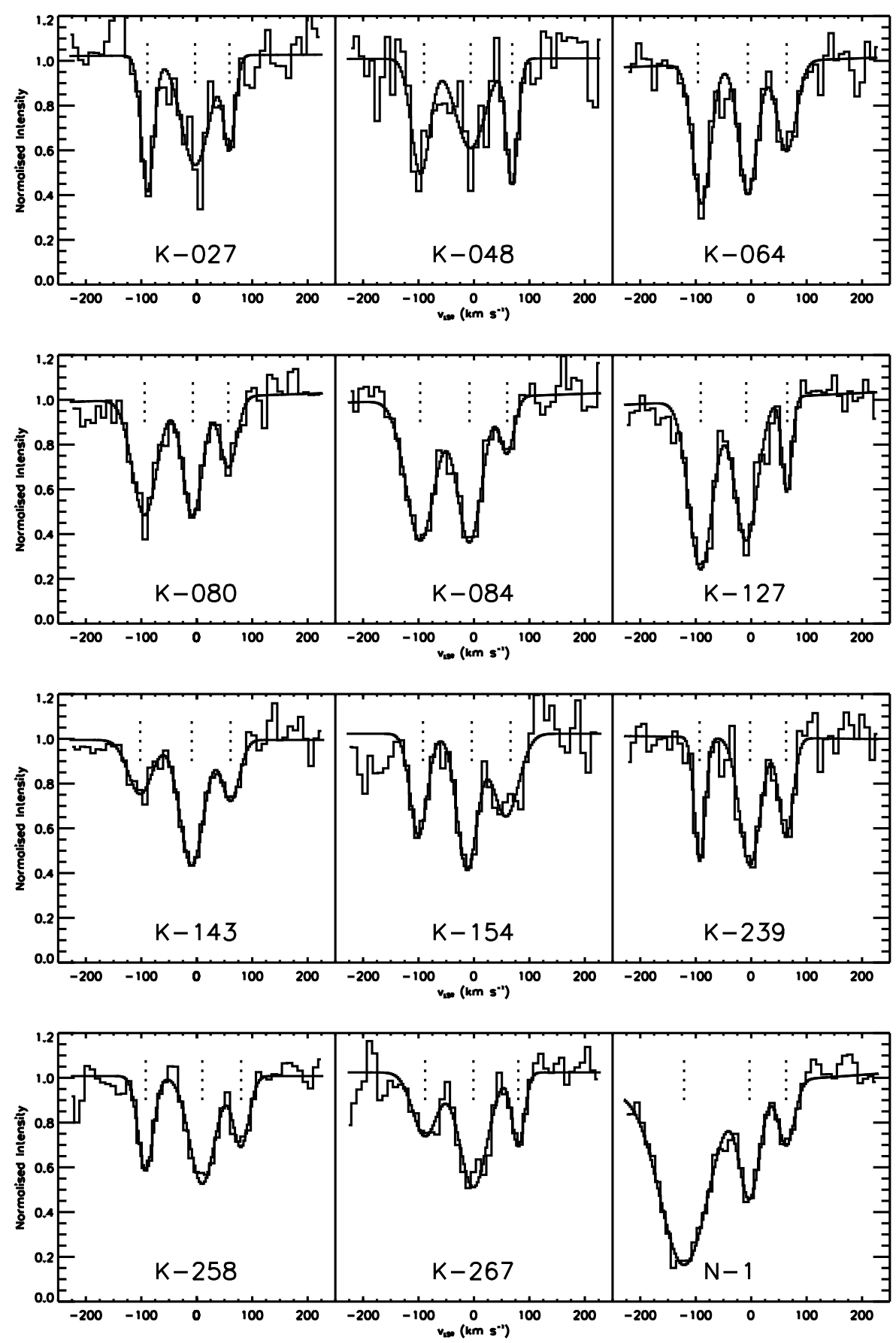

Fig. 2. Observed spectra (histograms) and their respective fits (smooth lines) of the Ca II $\mathrm{K}(\lambda \sim 3933.66 \AA$ ) line for the selected M 15 cluster stars. The stellar component is at $v_{\mathrm{LSR}} \sim-100 \mathrm{~km} \mathrm{~s}^{-1}$, the low- and high-velocity interstellar components are at velocities $\sim 0$ and $70-80 \mathrm{~km} \mathrm{~s}^{-1}$ respectively

for a sample of eight stars in a $\sim 25^{\prime \prime}$ strip (i.e. the stars between the dotted lines in Fig. 1). For the relative velocities $v_{\text {rel }}$, we have a taken an uncertainty of $\pm 5 \mathrm{~km} \mathrm{~s}^{-1}$. Since all the measured velocities do not have uncertainties greater than $5 \mathrm{~km} \mathrm{~s}^{-1}$, and this value corresponds to half a pixel, it seems quite reasonable to assign this uncertainty to $v_{\text {rel }}$. On this small scale, the equivalent widths vary randomly from 0.05 to $0.20 \AA$, and the velocities from 65 to
$80 \mathrm{~km} \mathrm{~s}^{-1}$, with mean values of $\sim 0.12 \AA$ and $70 \mathrm{~km} \mathrm{~s}^{-1}$. An inspection of Fig. 4 reveals that any variations in these quantities over small scales are probably on the order of or less than the measurement errors. Higher quality data are needed to quantify the magnitude of these variations. In particular, closely spaced pairings of sight lines such as the K-064, K-084, K-143 combination (identified in Fig. 4) provide the possibility of measuring HVC absorption over 

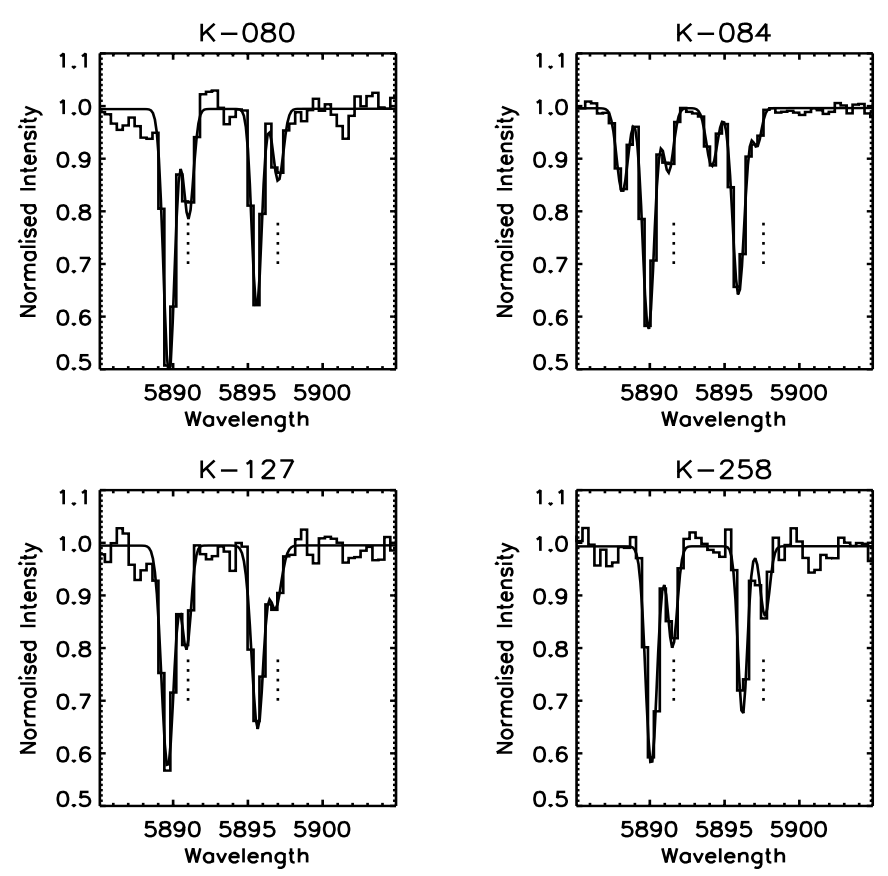

Fig. 3. Spectra (histograms) and their respective fits (smooth lines) of the $\mathrm{NaI} \mathrm{D}_{1}$ and $\mathrm{D}_{2}(\lambda \sim 5891.91$ and $5889.95 \AA)$ lines for the selected M 15 cluster stars. The HVC position is indicated by dashed lines

scales of $50^{\prime \prime}$. These data could then be combined with high spatial resolution $\mathrm{H}$ I maps to provide reliable estimates of $\mathrm{H}$ I column density at similar locations. In this work, we have shown that absorption is detectable across the face of the cluster and that many of these sight lines are suitable for such studies.

Such observations will also allow us to investigate the fractal structure of the HVC on scale-sizes of a few arcseconds. According to Vogelaar \& Wakker (1994) all structural details will disappear if the resolution is not sufficient to resolve individual cloudlets. Also the cloudlets within HVCs have been suggested as sites for star formation in the halo via cloudlet-cloudlet collisions (Dyson \& Hartquist 1983). This mechanism has been invoked to explain the existence of several early-type stars far from the plane of the Galaxy, whose kinematics and $z$-distances are incompatible with disc ejection (Keenan 1992). However these computations are extremely model-dependent (see, for example, Christodoulou et al. 1997) and require as input detailed information on the sizes, number density and physical conditions of HVC cloudlets, which are currently very uncertain (Wakker \& van Woerden 1997), and that our future observations should provide.

\section{Conclusions}

We have presented low resolution spectra of the sightlines towards the M 15 globular cluster in both the CaII K and NaI D interstellar lines, and have identified high-
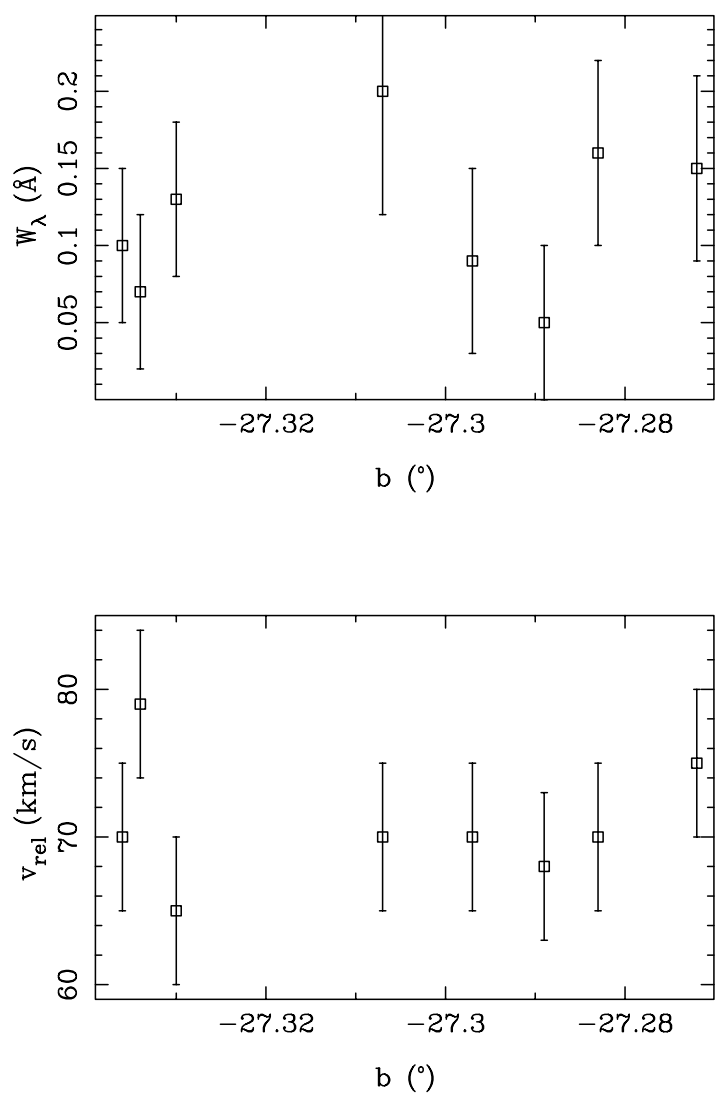

Fig. 4. Ca II equivalent widths and velocities of the HVC as a function of galactic latitude $b$ for the subset of stars indicated in Fig. 1

velocity clouds with velocities between $\sim 60-80 \mathrm{~km} \mathrm{~s}^{-1}$. The present data suggest that velocity and equivalent width variations may exist on small scales, but higher quality data are necessary to confirm this. Together with higher quality absorption line observations, high resolution $\mathrm{H}$ I maps of the region would provide detailed information on the substructure and size distribution of cloudlets within the HVCs.

Acknowledgements. NL holds a postgraduate studenship from the European Social Fund and Northern Ireland Development for Research. WRJR and RSIR acknowledge financial support from the UK Particle and Astronomy Research Council.

\section{References}

Battistini P., Bregoli G., Fusi Pecci F., Lolli M., Epps Bingham E.A., 1985, A\&AS 61,487

Buananno R., Buscema G., Corsi C.E., Iannicola G., Fusi Pecci F., 1983, A\&AS 51, 83

Christodoulou D.M., Tohline J.E., Keenan F.P., 1997, ApJ 468, 810

Dull J.D., Cohn H.N., Lugger P.M., et al., 1997, ApJ 481, 267

Durrel P.R., Harris W.E., 1993, AJ 105, 4 
Dyson J.E., Hartquist T.W., 1983, MNRAS 203, 1233

Howarth I.D., Murray J., Mills D., Berry D.S., 1996, STARLINK User Note SUN 50, Rutherford Appleton Laboratory/CCLRC

Keenan F.P., 1992, QJRAS 33, 325

Kennedy D.C., Bates B., Keenan F.P., et al., 1998, MNRAS (in press)

Langer G.E., Prosser C.F., Sneden C., 1990, AJ 100, 1

Little J.E., Dufton P.L., Keenan F.P., Conlon E.S., Davies R.D., 1994, ApJ 427, 267

Mihalas D., Binney L., 1981, Galactic Astronomy, 2nd edition.
San Francisco: Freeman

Morris R., Privett G., 1995, Starlink User Note SUN 79, Rutherford Appleton Laboratory/CCLRC

Ryans R.S.I., Sembach K.R., Kenaan F.P., 1996, A\&A 314, 609

Sembach K.R., 1995, ApJ 445, 314

Vogelaar M.G.R., Wakker B.P., 1994, A\&A 291, 557

Wakker B.P., van Woerden H., 1997, ARA\&A 35, 217

Wallace P.T., Clayton C., 1996, RV, STARLINK User Note SUN 78, Rutherford Appleton Laboratory/CCLRC 